\title{
Space-Time Correlations
} and Dynamic Coupling in Turbulent Flows

\section{Guowei He, ${ }^{1}$ Guodong Jin, ${ }^{1}$ and Yue Yang ${ }^{1,2}$}

\author{
${ }^{1}$ State Key Laboratory of Nonlinear Mechanics, Institute of Mechanics, Chinese Academy of \\ Sciences, Beijing 100190, China; email: hgw@Inm.imech.ac.cn \\ ${ }^{2}$ State Key Laboratory of Turbulence and Complex Systems and Center for Applied Physics \\ and Technology, College of Engineering, Peking University, Beijing 100871, China
}

Annu. Rev. Fluid Mech. 2017. 49:51-70

First published online as a Review in Advance on May 23, 2016

The Annual Review of Fluid Mechanics is online at fluid.annualreviews.org

This article's doi:

10.1146/annurev-fluid-010816-060309

Copyright (c) 2017 by Annual Reviews. All rights reserved

\section{Keywords}

Eulerian and Lagrangian time correlations, large-eddy simulation, Taylor's frozen-flow hypothesis, time-accurate subgrid-scale model, turbulence-generated noise, particle-laden turbulence

\begin{abstract}
Space-time correlation is a staple method for investigating the dynamic coupling of spatial and temporal scales of motion in turbulent flows. In this article, we review the space-time correlation models in both the Eulerian and Lagrangian frames of reference, which include the random sweeping and local straining models for isotropic and homogeneous turbulence, Taylor's frozen-flow model and the elliptic approximation model for turbulent shear flows, and the linear-wave propagation model and swept-wave model for compressible turbulence. We then focus on how space-time correlations are used to develop time-accurate turbulence models for the large-eddy simulation of turbulence-generated noise and particle-laden turbulence. We briefly discuss their applications to two-point closures for Kolmogorov's universal scaling of energy spectra and to the reconstruction of space-time energy spectra from a subset of spatial and temporal signals in experimental measurements. Finally, we summarize the current understanding of space-time correlations and conclude with future issues for the field.
\end{abstract}


LES large-eddy simulation

PIV particle image velocimetry

\section{EDQNM}

eddy-damped quasi-normal

Markovian

\section{RANS}

Reynolds-averaged Navier-Stokes

\section{INTRODUCTION}

Space-time correlations are fundamental for understanding the dynamic coupling between the spatial and temporal scales of motion in turbulent flows. Such correlations quantify how turbulent fluctuations at one location and one instant covary with those at another location and another instant, and thus describe the dynamic behaviors of turbulent fluctuations across spatial and temporal scales. Over the past few decades, space-time correlations have been used to provide the timescales necessary for two-point closure methods, to develop time-accurate turbulence models for large-eddy simulation (LES), and to analyze spatiotemporal data from experimental measurements. This method is widely applicable to many aspects of turbulence research.

The first model of space-time correlations was based on Taylor's frozen-flow hypothesis. In his famous paper "The Spectrum of Turbulence," Taylor (1938, p. 476) originally proposed that "the sequence of changes in $u$ (velocity fluctuations) at the fixed point are simply due to the passage of an unchanging pattern of turbulent motions over the period," where the passage speed is the velocity of the wind stream. Therefore, a space-time correlation can be approximately expressed by a time correlation and a passage speed. This result has been broadly used to calculate spatial energy spectra in hot-wire measurements (del Álamo \& Jiménez 2009) and has recently been used to reconstruct space-time energy spectra in particle image velocimetry (PIV) measurements (de Kat \& Ganapathisubramani 2015). Taylor's model also provides insights into the spatiotemporal dynamics of turbulent flows, such as the dominant convection process (Heskestad 1965) and propagation speeds of coherent structures (Kim \& Hussain 1993). However, this model suffers from many limitations (Moin 2009), including low turbulence intensities and weak shear rates, and has been corrected in different circumstances.

Eulerian and Lagrangian time correlations are essential to the statistical theory of turbulence. The Kolmogorov phenomenological theory developed in 1941 predicts the universal scaling $-5 / 3$ of spatial energy spectra for isotropic and homogeneous turbulence (Frisch 1995). However, the analytical derivation of this universal scaling from the Navier-Stokes equations remains a challenge. Several deductive approaches have been developed, such as direct interaction approximation (Kraichnan 1959) and the eddy-damped quasi-normal Markovian (EDQNM) (Orszag 1977). In these approaches, space-time correlation models are needed to provide the characteristic timescales to close the energy spectra equations. If Eulerian timescales are used, the resulting scaling is $-3 / 2$, whereas if Lagrangian timescales are used, the resulting Kolmogorov scaling is $-5 / 3$. The Eulerian time correlation is associated with the random sweeping process, whereas the Lagrangian time correlation is associated with the local straining process (Kraichnan 1964). Both random sweeping and local straining can be found in turbulent flows and represent two distinct decorrelation processes.

The growing application of LES to turbulence-generated noise and particle-laden turbulence requires LES to correctly predict space-time correlations in Eulerian and Lagrangian frames of reference. In aeroacoustics, the intensity of the far-field noise radiated by turbulent flows is crucially dependent on Eulerian space-time correlations in terms of the Lighthill acoustic analogy (Lighthill 1952). In particle-laden turbulence, particle dispersions are essentially determined by Lagrangian time correlations. Accordingly, the accurate prediction of space-time correlations is becoming an important requirement for LES. However, this requirement cannot be met through the use of the Reynolds-averaged Navier-Stokes (RANS) approach. The RANS approach is designed to capture spatial statistics of turbulent flows such as the mean velocity and spatial correlations, but temporal statistics are not considered. In practice, the RANS equations are derived only for the mean velocities and spatial correlations. Time correlations are the unknown component in the RANS equations. In LES, both spatial and temporal motions at large scales are available. Therefore, LES 
provides new opportunities for the accurate prediction of temporal statistics. However, previous studies have focused on spatial statistics and did not benefit from the full potential of LES.

This article is organized as follows. In Section 2, we review Eulerian and Lagrangian spacetime correlation models. These models include the random sweeping model and local straining model for isotropic and homogeneous turbulence, Taylor's frozen-flow model and the elliptic approximation (EA) model for turbulent shear flows, and the linear-wave propagation model and swept-wave model for compressible turbulence. In Section 3, we discuss the application of spacetime correlations for the development of time-accurate subgrid-scale (SGS) models for LES of turbulence-generated noise and particle-laden turbulence. Finally, we summarize the present state of space-time correlations and raise future issues for the field. For the sake of brevity, this review focuses on recent progress in studies of space-time correlations. For readers interested in the relevant topics, we suggest recent reviews on the two-point closure approach (Smith \& Woodruff 1998, Cambon \& Scott 1999, Zhou 2010) and the conversion between temporal and spatial signals obtained from experimental measurements (Lohse \& Xia 2010, Smits et al. 2011, Wallace 2014). Meneveau \& Katz (2000) provided an excellent review on SGS models for LES. Some key aspects of the LES of turbulence-generated noise are reviewed by Wang et al. (2006). Updated reviews on particle-laden turbulence are provided by Balachandar \& Eaton (2010) and Fox (2012).

\section{SPACE-TIME CORRELATIONS IN TURBULENT FLOWS}

\subsection{Space-Time Correlations in Isotropic Turbulence}

The random sweeping hypothesis proposes that small-scale eddies are randomly swept by largescale, energy-containing eddies without significant distortion (Kraichnan 1964) (see the sidebar The Random Sweeping and Local Straining Hypotheses). This hypothesis implies a simple model equation for Eulerian velocities:

$$
\frac{\partial \hat{u}_{j}(\mathbf{k}, t)}{\partial t}+i(\mathbf{k} \cdot \mathbf{v}) \hat{u}_{j}(\mathbf{k}, t)=0
$$

where $\hat{u}_{j}$ is the Fourier mode of the velocity fluctuations, $\mathbf{k}$ is the wave-number vector of magnitude $k, \mathbf{v}$ is the constant velocity of a Gaussian distribution over all realizations, and $i$ is the imaginary unit. The symbols with hats denote the Fourier modes, such as $\hat{u}_{j}(\mathbf{k}, t)$, which is the Fourier mode of $u_{j}(\mathbf{x}, t)$. The solution to this model equation gives the time correlations of the velocity modes:

$$
\begin{aligned}
\hat{R}_{E}(k, \tau) & \equiv\left\langle\hat{u}_{j}(\mathbf{k}, t) \hat{u}_{j}(-\mathbf{k}, t+\tau)\right\rangle \\
& =E(k) \exp \left(-\frac{1}{2} k^{2} V^{2} \tau^{2}\right),
\end{aligned}
$$

where $V$ is the root mean square of the velocity fluctuations, $E(k)$ denotes the spatial energy spectra, and the angle brackets denote an ensemble average. Space-time correlations can be equivalently

\section{THE RANDOM SWEEPING AND LOCAL STRAINING HYPOTHESES}

The Eulerian decorrelation is different from the Lagrangian decorrelation: The former is dominated by the sweeping of energy-containing eddies, whereas the latter is dominated by the straining of local eddies. Therefore, the Eulerian frequency spectra scale as $-5 / 3$, and the Lagrangian frequency spectra scale as -2 , with these results supported by the direct numerical simulation of isotropic turbulence. 
PDF probability density function transformed to modal time correlations, and vice versa. The random sweeping model indicates that the Eulerian decorrelation timescales are $(k V)^{-1}$.

The local straining hypothesis proposes that the Lagrangian time correlations are dominated by local straining (Kraichnan 1968). This can be inferred from the kinematic equation for Lagrangian velocities:

$$
\frac{\partial v_{j}^{\ell}(\mathbf{x}, s \mid t)}{\partial s}+\mathbf{u}(\mathbf{x}, s) \cdot \nabla v_{j}^{\ell}(\mathbf{x}, s \mid t)=0
$$

where $v_{j}^{\ell}$ is the velocity, measured at time $t$, of the fluid particle located at position $\mathbf{x}$ at time $s$. The Lagrangian velocity $v_{j}^{\ell}$ is simply advected by the Eulerian velocity $\mathbf{u}$, which is governed by the Navier-Stokes equations. Lagrangian closure approaches, such as the Lagrangian history direct interaction approximation (Kraichnan 1968) and Lagrangian renormalized approximation (Kaneda 1981), show that Lagrangian time correlations are dominated by the local straining timescale (Gotoh et al. 1993):

$$
\left(\int_{0}^{k} p^{2} E(p) \mathrm{d} p\right)^{-1 / 2}
$$

where the Lagrangian time correlations are defined by

$$
\hat{R}_{L}(k, \tau) \equiv\left\langle\hat{v}_{j}^{\ell}(\mathbf{k}, s \mid t) \hat{v}_{j}^{\ell}(-\mathbf{k}, s \mid t+\tau)\right\rangle .
$$

Tennekes (1975) suggested that the Eulerian frequency spectra are dominated by random advection, whereas Lagrangian frequency spectra are determined by the inertial timescales. As a result, the Eulerian frequency spectra scale as $\omega^{-5 / 3}$ and Lagrangian frequency spectra scale as $\omega^{-2}$. This implies that the rate of Eulerian decorrelation is higher than that of Lagrangian decorrelation, which is consistent with the random sweeping and local straining hypotheses (Chen \& Kraichnan 1989, Rubinstein \& Zhou 1999) and confirmed by the mutual cancellation between local and convective accelerations (Tsinober et al. 2001).

The decorrelation timescales are crucial to the successes of two-point closures such as EDQNM (Orszag 1977). In the EDQNM approach, the energy spectra depend on the forms of eddy damping, which are determined by the decorrelation timescales. If Eulerian decorrelation timescales are used, a scaling of $-3 / 2$ is obtained, which is inconsistent with the Kolmogorov scaling. If Lagrangian decorrelation timescales are used instead, the Kolmogorov scaling of $-5 / 3$ is recovered. To deduce the Lagrangian timescales, Kraichnan (1968) developed the Lagrangian history direct interaction approximation. Later, Kaneda (1981) constructed the Lagrangian renormalization approximation to derive the timescales. Recently, Bos \& Bertoglio (2013) proposed a self-consistent framework for velocity-position cross correlations to determine the timescales.

Relating Lagrangian and Eulerian time correlations remains an important but unresolved problem in the turbulence community. In some sense, this is equivalent to the turbulence closure problem. Corrsin (1959) proposed the well-known independent hypothesis that the probability density functions (PDFs) of relative separations are independent of the PDFs of the Eulerian velocity field. This hypothesis circumvents the main difficulties of relating Lagrangian correlations with Eulerian ones and yields an approximate relationship between Lagrangian and Eulerian statistics. Later on, this independent hypothesis was shown to be equivalent to the first-order truncation of the renormalized perturbation expansion (Kraichnan 1977). Homann et al. (2009) proposed an exact relationship between the PDFs of Lagrangian and Eulerian velocity increments. This relationship explains how intermittency in Lagrangian velocity increments is linked with Eulerian ones through the transition PDFs. The link between Eulerian and Lagrangian velocity increments has been phenomenologically established in the framework of multifractal theory (Borgas 1993, Chevillard et al. 2003, Biferale et al. 2004, Xu et al. 2006). The Smith-Hay model expresses the 
Lagrangian velocity correlation using Eulerian velocity correlations and Taylor's frozen-flow hypothesis (Smith \& Hay 1961). This model is the first approximation to iso-correlation contours. The EA model for Lagrangian space-time correlations accounts for the flow distortion using second-order approximation to the iso-correlation contours (He et al. 2009).

\subsection{Taylor's Frozen-Flow Model}

In his famous paper "The Spectrum of Turbulence," Taylor (1938) hypothesized that the spatial patterns of turbulent motions are carried past a fixed point at the convection velocity without changing significantly. In other words, the spatial patterns move as the frozen flow moves. The convection velocity was taken as the speed of the wind stream in Taylor's original paper and is usually taken as the local average flow velocity. According to Taylor's frozen-flow hypothesis, if the streamwise velocity component at location $\mathbf{x}=\left(x_{1}, x_{2}, x_{3}\right)$ and time $t$ is $u(\mathbf{x}, t)$, then the velocity $u(\mathbf{x}+\mathbf{r}, t+\tau)$ at the downstream location $\mathbf{x}+\mathbf{r}=\left(x_{1}+r, x_{2}, x_{3}\right)$ and the later time $t+\tau$ can be approximately expressed by

$$
u(\mathbf{x}+\mathbf{r}, t+\tau)=u(\mathbf{x}+\mathbf{r}-\mathbf{U} \tau, t),
$$

where $\mathbf{U}=(U, 0,0)$ is the convection velocity and $U$ is constant. Hence, the space-time correlation is given by

$$
R(r, \tau) \equiv\langle u(\mathbf{x}, t) u(\mathbf{x}+\mathbf{r}, t+\tau)\rangle=R(r-U \tau, 0) .
$$

Taylor's model expresses the space-time correlations using the space correlations and a linear transformation $r-U \tau$. This linear transformation is the consequence of the frozen-flow hypothesis and implies that the space-time correlations never decay for any separations $r$ or $\tau$ on the characteristic lines $r-U \tau=$ const. (see Figure $\mathbf{1} \boldsymbol{a}, \boldsymbol{b}$ ), which violates the physical nature of correlation functions (see the sidebar Taylor's Frozen-Flow Hypothesis and Iso-Correlation Contours). Lin (1953) showed that Taylor's model is a good approximation if the turbulent intensity is very low; however, there is no general justification for extending Taylor's hypothesis to the case of shear flows. Lumley (1965) explored the possible mechanisms for invalidating Taylor's model in turbulent shear flows and proposed the corrected forms for small-scale motions. Applications of Taylor's frozen-flow hypothesis to the time-to-space conversion in experimental measurements have been discussed in recent review articles (Lohse \& Xia 2010, Smits et al. 2011, Wallace 2014). In this subsection, we focus on the results related to space-time correlations.

The convection velocity is the only parameter in Taylor's model. Davies et al. (1963) experimentally observed that the convection velocity is not equal to the local mean velocity in the mixing region of a round jet. Wills (1964) examined the effective convection velocity defined by space-time correlations and used space-time energy spectra to obtain a wavelength-dependent convection velocity. However, he argued that there exists an overall convection velocity that dominates the space-time correlations. It is extremely difficult to experimentally measure space-time correlations or frequency-wave-number spectra. To circumvent this difficulty, del Álamo \& Jiménez

\section{TAYLOR'S FROZEN-FLOW HYPOTHESIS AND ISO-CORRELATION CONTOURS}

Taylor's model implies that the iso-correlation contours are straight lines. The elliptic approximation model implies second-order approximations to the iso-correlation contours, which can account for the decorrelation induced by convection and shearing of mean flows and random sweeping of velocity fluctuations. 

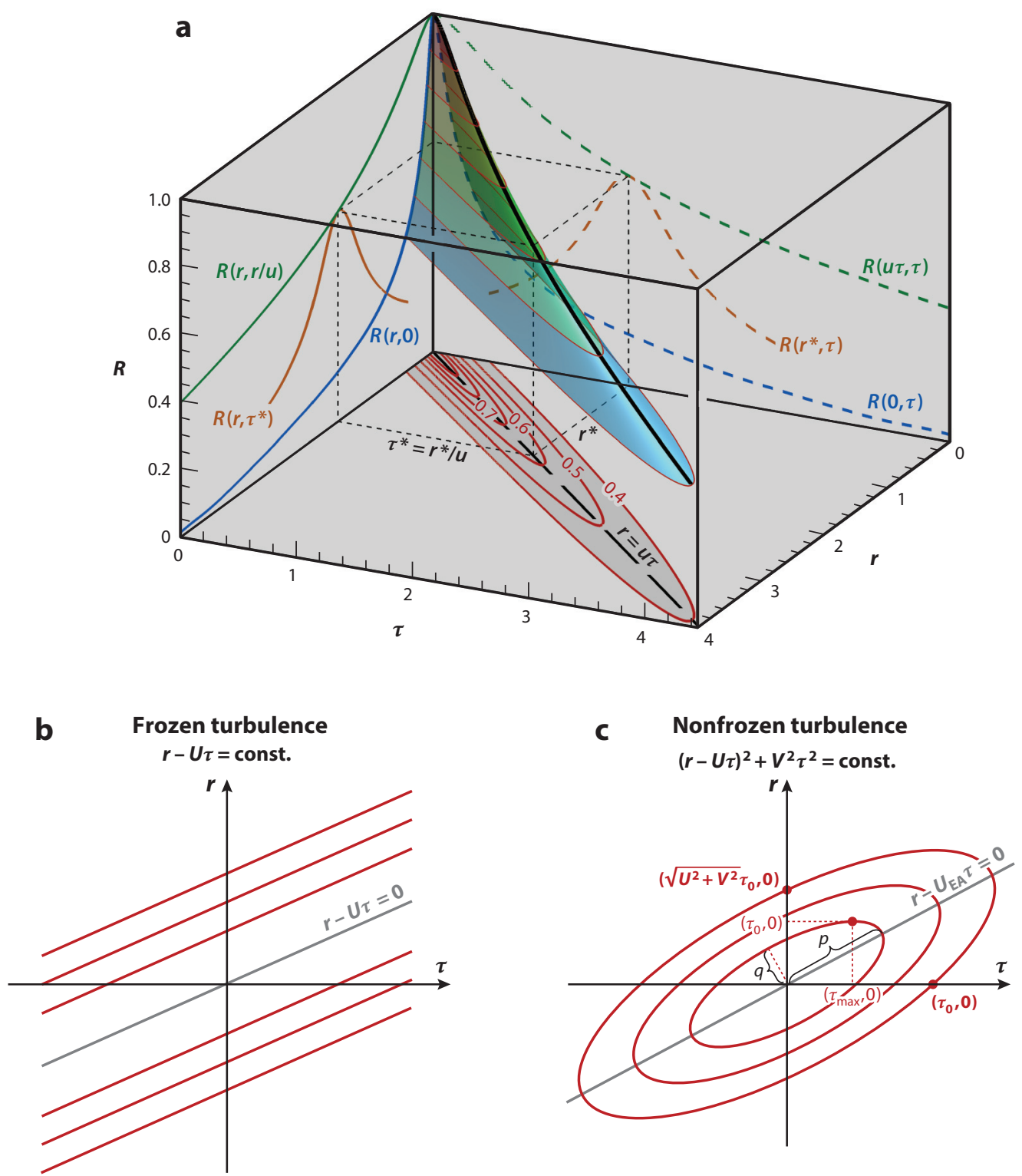

Figure 1

(a) The space-time correlation of the streamwise velocity $u$ at $y^{+}=92$ in the logarithmic layer, from the direct numerical simulation of turbulent channel flows at $R e_{\tau}=550$, plotted as a function of space separation $r$ and time delay $\tau$. The correlation decays most slowly along the ridge line (black solid line); the shadow-code iso-correlation contours on the $(r, \tau)$ plane are the closed lines with a preferential direction, aligned with the projection of the ridge line: $r=U \tau$ ( $U$ is a propagation velocity). The projections of the ridge line onto the planes $(r, R)$ (green solid line) and $(\tau, R)$ (green dashed line) are also plotted. The spatial correlation (blue solid line) and time correlation (blue dashed line) are shown as references. The spatial correlation $R\left(r, \tau^{*}\right)$ (brown solid line) for a fixed time delay $\tau^{*}$ and the time correlation $R\left(r^{*}, \tau\right)$ (brown dashed line) for a fixed space separation $r^{*}$ are also illustrated. $(b, c)$ Schematic diagrams for the iso-correlation contours of $(b)$ frozen and $(c)$ nonfrozen turbulence. Panel $b$ corresponds to Taylor's model, which implies that the contours are straight lines, whereas panel $c$ corresponds to the elliptic approximation (EA) model, which implies that the contours are elliptic curves. $U_{\mathrm{EA}}$ is the propagation velocity in the EA model. 
(2009) utilized the temporal information of velocity and its spatial or temporal derivatives. The obtained convection velocity is scale dependent and can be used to correct the scalings of energy spectra. For spatially developing flows, the heterogeneity of streamwise velocities is problematic for the calculation of spatial energy spectra. Renard \& Deck (2015) derived a new approach to estimate the frequency-dependent convection velocity from the temporal signals of the velocity and its local spatial derivatives. Recently, de Kat \& Ganapathisubramani (2015) emphasized that the convection velocity is a distribution of phase velocities and developed a time-to-space mapping approach to reconstruct full space-time energy spectra.

Space-time correlations have been used to estimate the propagation speeds of turbulent fluctuations. Kim \& Hussain (1993) estimated propagation speeds of velocity, vorticity, and pressure fluctuations using direct numerical simulation (DNS) data from turbulent channel flows. The propagation speeds are approximately equal to the local mean velocities and are scale independent in the outer region; however, they are increasingly larger than the local mean velocities and become scale dependent as they approach the wall. Zaman \& Hussain (1981) estimated the convection velocities of coherent structures in the near fields of circular jets and showed that Taylor's model can be applied to a single coherent structure but misrepresents multiple coherent structures.

The following generalized form of the convection equation was proposed by Heskestad (1965) to better understand the dynamics of Taylor's frozen-flow hypothesis:

$$
\frac{\partial u_{i}}{\partial t}+U \frac{\partial u_{i}}{\partial x}+u_{j} \frac{\partial U}{\partial x_{j}}+u_{j} \frac{\partial u_{i}}{\partial x_{j}}=0
$$

where $U$ and $u_{i}$ denote the convection velocity and fluctuating velocity components, respectively, in the coordinate direction $x_{j}=x, y, z$. The temporal evolution of turbulent fluctuations (first term) is determined by the convection (second term), shearing (third term), and nonlinear terms (fourth term). Taylor's model is simply the solution of Equation 8 if the convection velocity is constant and the nonlinear term is ignored; the random sweeping model is the solution of Equation 8 if the convection velocity vanishes and the nonlinear term is treated as random sweeping. Loucks \& Wallace (2012) compared the generalized form with the original form of Taylor's model in turbulent boundary layer and plane mixing layer flows. Data from LES (Piomelli et al. 1989) and DNS (Geng et al. 2015) of turbulent channel flows are also used to examine the general form in comparison with the full Navier-Stokes equations. These results show that the temporal derivatives of the velocity fluctuations can be reasonably approximated by the product of the optimal convection velocity and the spatial gradients of the velocity fluctuations. However, the additional terms that are not responsible for Taylor's model diminish the validity of this model.

\subsection{The Elliptic Approximation Model in Turbulent Shear Flows}

The EA model proposes that space-time correlations in turbulent shear flows can be expressed by space correlations $R(r, 0)$ and two characteristic velocities $U$ and $V$ :

$$
R(r, \tau)=R\left[\sqrt{(r-U \tau)^{2}+V^{2} \tau^{2}}, 0\right],
$$

where $U$ characterizes the convection of flow patterns and $V$ characterizes the distortion of flow patterns (He \& Zhang 2006, Zhao \& He 2009). The EA model represents the decorrelation of small-scale eddies in turbulent shear flows: Small-scale eddies are convected by mean flows and are distorted by the shearing of mean flows and the sweeping of energy-containing eddies in fluctuating velocity fields. In Taylor's model, the decorrelation is determined solely by the convection velocity, which describes the displacements of small-scale eddies due to mean flows. In the
DNS direct numerical simulation 


\section{RBC}

Rayleigh-Bénard convection
Kraichnan-Tennekes model, the decorrelation is mainly determined by the random sweeping velocity, which describes the sweeping of small-scale eddies by energy-containing eddies (Kraichnan 1964, Tennekes 1975). Consequently, the EA model manifests the coupled effects of convection and random sweeping. This model takes Taylor's model and the Kraichnan-Tennekes random sweeping model as two extreme cases at vanishing characteristic velocities $U$ and $V$.

The EA model is developed through a successive approximation to the iso-correlation contours. Figure 1 shows the space-time correlation contours from DNS of turbulent channel flows at $R e_{\tau}=550$ (Geng et al. 2015), including the iso-correlation contours defined by Taylor's and EA models. The following two aspects are worth highlighting. First, the EA model is the second-order approximation to iso-correlation contours, whereas Taylor's model is the linear approximation to the contours. Taylor's model implies that the correlations remain constant along the characteristic lines $r-U \tau=$ const. However, this implication is inconsistent with the physical nature of correlation functions, which should decay with increasing spatial or temporal separation. Second, the contours of the space-time correlations over separations in the inertial range share a preferential direction $U$ and an aspect ratio $p / q \simeq V$, which is consistent with Taylor's hypothesis of the dominant convection and with Kolmogorov's similarity hypothesis.

The EA model can be used to reconstruct space-time correlations from temporal correlations (He et al. 2010). If the time correlations $R_{1}(0, \tau)$ at a fixed point and $R_{2}\left(r_{0}, \tau\right)$ at two points separated a distance $r_{0}$ are known, we can use the EA model to calculate the space-time correlations for a particular range of spatial separations (see Figure 1c):

$$
R(r, \tau)=R_{1}\left[0, \tau_{E}(r, \tau)\right],
$$

where $\tau_{E}(r, \tau)=\sqrt{(r-U \tau)^{2}+(V \tau)^{2}} / \sqrt{U^{2}+V^{2}}$. The parameters $U$ and $V$ can be estimated as follows. First, find $\tau_{\max }$ from the known $R_{2}\left(r_{0}, \tau\right)$ to maximize $R_{2}\left(r_{0}, \tau\right)=R\left[0, \tau_{E}\left(r_{0}, \tau\right)\right]$, which requires $\partial R_{2} / \partial \tau=\left(\partial R / \partial \tau_{E}\right)\left(\partial \tau_{E} / \partial \tau\right)=0$ at $\left(r_{0}, \tau_{\max }\right)$ and thus $\left(U^{2}+V^{2}\right) \tau_{\max }=U r_{0}$. Second, find $\tau_{0}$ from the known $R_{1}(0, \tau)$ such that $R_{1}\left(0, \tau_{0}\right)=R_{2}\left(r_{0}, 0\right)$, leading to $r_{0}^{2}=\left(U^{2}+V^{2}\right) \tau_{0}^{2}$. If the space-time correlations for multiple spatial separations are known, the parameters $U$ and $V$ can be averaged over multiple values. This conversion at $\tau=0$ yields an approximation of the spatial correlations via the temporal ones.

The EA model has been tested using DNS data for turbulent channel flows at the low Reynolds number of $R e_{\tau}=180$ (Zhao \& He 2009). The space-time correlations collapse to a universal form in both the wall region and outer layer with the time separation defined from the EA model, whereas this was only true in the outer layer with Taylor's model. Zhou et al. (2011) were the first to systematically validate the EA model for velocity fields in turbulent Rayleigh-Bénard convection (RBC). Their experimental results revealed that the EA model is valid for velocity fields in regions near the sidewall and central region of the cell, where Taylor's model does not hold. He et al. (2010) experimentally demonstrated that the EA model is also valid for local temperature fluctuations in turbulent RBC. Hogg \& Ahlers (2013) examined the EA model for space-time correlations of shadowgraph images in RBC and determined the Reynolds numbers in the GrossmannLohse model. Wang et al. (2014) used PIV data of turbulent boundary layers to validate the EA model.

At the Max Planck Institute for Dynamics and Self-Organization and the University of California, Santa Barbara, He et al. (2012b, 2014, 2015) extensively tested the validity of the EA model in turbulent RBC, where Taylor's frozen-flow hypothesis is not valid because of large fluctuations (Lohse \& Xia 2010). Their experimental results verify that the temperature correlation contours present similar elliptic shapes and the EA model leads to the collapse of all space-time correlations on the normalized space and time separations. Using the EA model, they unambiguously determined those two parameters $U$ and $V$ and the corresponding Reynolds numbers. The 
dependence of the Reynolds numbers on the Rayleigh numbers and Prandtl numbers in their experiments is consistent with the Grossmann-Lohse model (Grossmann \& Lohse 2001) and provides landmark evidence of the transition to the RBC ultimate state, predicted by Kraichnan (1962) (He et al. 2016).

Wilczek \& Narita (2012) proposed a simple equation for the space-time energy spectra. This equation consists of both constant and Gaussian random convection and is given by

$$
\frac{\hat{u}_{j}(\mathbf{k}, t)}{\partial t}+i[\mathbf{k} \cdot(\mathbf{U}+\mathbf{v})] \hat{u}_{j}(\mathbf{k}, t)=0
$$

which is the combination of Taylor's frozen-flow hypothesis and the Kraichnan-Tennekes random sweeping hypothesis. The solution yields a model for the space-time energy spectra:

$$
E(\mathbf{k}, \omega)=\frac{E(\mathbf{k})}{\sqrt{2 \pi k^{2} V^{2}}} \exp \left[\frac{-(\omega-\mathbf{k} \cdot \mathbf{U})^{2}}{2 k^{2} V^{2}}\right] .
$$

This model has the form of the product of a wave-number energy spectrum and a Gaussian frequency spectrum. It demonstrates that the Doppler shift is induced by the constant mean velocity and the frequency broadening is induced by the random sweeping. This model has been further parameterized in the logarithmic layer of wall-bounded turbulence and is in good agreement with LES data of fully rough-wall turbulent channel flows (Wilczek et al. 2015). The space-time correlations obtained from the energy spectra model lead to the approximately elliptic contours of self-similarity, which provides theoretical justification for the EA model.

\subsection{Space-Time Correlations in Compressible Turbulence}

Lee et al. (1992) developed a linear-wave propagation model for space-time correlations in compressible turbulence considering the solenoidal and dilatation modes of velocity fluctuations. DNS of spatially evolving turbulence shows that space-time correlations of solenoidal modes exhibit the same properties as incompressible turbulence. However, the space-time correlations of dilatational modes contrast with those of incompressible turbulence because acoustic waves propagate at the local sound speed relative to moving fluids. Based on the acoustic properties of dilatation modes, they proposed a linear-wave equation in a moving frame, which results in the linear-wave propagation model. This model predicts the main characteristics of space-time correlations of dilatation modes, namely, that the well-correlated regions oscillate in a preferential direction.

Li et al. (2013) generalized the linear-wave propagation model by including random sweeping effects. They argued that dilatation modes are swept by energy-containing eddies as they propagate at the speed of sound relative to the moving fluid. Therefore, random convection is introduced into the linear-wave equation as

$$
\left(\frac{D^{2}}{D t^{2}}-\bar{a}^{2} \nabla^{2}\right) u_{j}^{d}=0
$$

where $\bar{a}$ is the local speed of sound, $u_{j}^{d}$ is the dilatation component, and

$$
\frac{D}{D t}=\frac{\partial}{\partial t}+\mathbf{v} \cdot \nabla
$$

This model equation leads to the swept-wave model for dilatation correlations:

$$
R_{D}(r, \tau)=\int_{0}^{\infty} \hat{R}_{D}(k, 0) \exp \left(-\frac{1}{2} V^{2} k^{2} \tau^{2}\right) \cos (k \bar{a} \tau) \frac{\sin [k(r-U \tau)]}{k(r-U \tau)} \mathrm{d} k .
$$


This model correctly predicts the decay of the space-time correlations of dilatational modes, thus obtaining good agreement with DNS of compressible-isotropic turbulence.

\section{TIME-ACCURATE SUBGRID-SCALE MODELING FOR LARGE-EDDY SIMULATIONS}

In LES, large-scale motions are explicitly resolved, which is not the case for small-scale motions. However, an SGS model is used to account for the effects of small-scale motions on large-scale motions. Conventional SGS models are constructed using energy budget equations so that a balance of the energy transfer between the resolved and unresolved scales can be achieved. For example, the eddy-viscosity SGS model acts as a sink to dissipate energy at unresolved scales. Therefore, this class of dissipative model can maintain the energy balance in LES and thus correctly predict energy spectra or, equivalently, spatial correlations. However, such models may not correctly predict space-time correlations because these are not determined by spatial correlations alone. In turbulent flows, the spatial and temporal scales are dynamically coupled so that spatiotemporal statistics are not fully determined by spatial statistics. Therefore, the accurate prediction of spatiotemporal statistics represents a new requirement for SGS modeling in LES. The objective of time-accurate SGS models is to accurately predict space-time correlations or, more generally, multi-time and multipoint statistics. This contrasts with the dissipative SGS models that are developed to reproduce single-time and multipoint statistics. Time-accurate SGS models not only represent theoretical interests but also have practical applications to turbulence-generated noise and particle-laden turbulence.

\subsection{Eulerian Space-Time Correlations for Large-Eddy Simulations of Turbulence-Generated Noise}

Eulerian space-time correlations are introduced as a diagnostic tool to evaluate the ability of SGS modeling to predict the temporal properties of turbulent flows. He et al. (2002) compared time correlations of velocity modes in stationary isotropic and homogeneous turbulence calculated via DNS with those calculated via LES using the spectral eddy-viscosity SGS model. This comparison revealed that the correlation functions from LES decay more slowly at all resolved scales and that both their integral scales and microscales are larger than those from DNS. The results indicating that LES with the eddy-viscosity SGS model overpredicts time correlations are confirmed by EDQNM analysis. The overpredictions are also found in decaying isotropic turbulence (He et al. 2004), rotating turbulence (Favier et al. 2010), turbulent channel flows (Guo et al. 2012), and compressible turbulent boundary layers (Martin 2005). Park et al. (2005) concluded that the class of deterministic SGS models would yield the slower decorrelation of space-time correlations in isotropic turbulence. Dong \& Sagaut (2008) showed that lattice Boltzmann SGS models underestimate the magnitudes of the modal time correlations for a range of wave numbers, similar to Navier-Stokes SGS models. Comparisons of dissipative SGS models in LES suggest that the dynamic Smagorinsky SGS model in conjunction with the multiscale LES procedure presents the most accurate predictions of space-time correlations (He et al. 2004).

The reason that eddy-viscosity SGS models overpredict space-time correlations is that these models do not consider random backscattering (see the sidebar Backscattering, Space-Time Correlation, and the Eddy-Viscosity Model). In LES, the SGS stress has two main functions: energy dissipation and random backscattering. The former removes energy from large scales, and the latter intermittently transfers energy to large scales (Piomelli et al. 1991). Random backscattering acts as a stochastic force that stirs large-scale motions and reduces their coherence. Eddy-viscosity 


\section{BACKSCATTERING, SPACE-TIME CORRELATION, AND THE EDDY-VISCOSITY MODEL}

Small-scale motions have two main effects on large-scale motions: energy dissipation and random backscattering. The former results in small-scale motions acting as sinks that absorb energy from large-scale motions, whereas the latter results in small-scale motions acting as sources that randomly transfer energy backward to large-scale motions, which reduces the correlation length scales of large-scale motions in both space and time. The eddy-viscosity model accounts for energy dissipation but ignores random backscattering. As a result, this model overpredicts space-time correlations in LES.

SGS models represent energy dissipation but omit random backscattering. As a result, these models generate more coherent flow structures and increase the spatial and time scales (Guo et al. 2012). This contrasts with LES of transitional flows at the early nonlinear stage, where the mean energy is transferred from small scales to large scales (Piomelli et al. 1991). The effects of SGS backscattering on eddy viscosities for passive and active scalars were recently investigated by O'Brien et al. (2014).

A straightforward approach to developing an SGS model for predicting space-time correlations is to add random components to the SGS model. Random forcing can compensate for the effects of random backscattering, as proposed by Bertoglio (1985), Leith (1990), and Mason \& Thomson (1992) for the predictability problem of turbulence and by Carati et al. (1995) for dynamic Smagorinsky models. Random fluctuations can be directly added to the eddy viscosity and thus generate a partial stochastic eddy viscosity. The stochastic eddy-viscosity model has been found to increase the decay rates of space-time correlations in comparison with the Smagorinsky SGS model (Marstorp et al. 2007). Another approach is to use structural SGS models (Clark et al. 1979, Bardina et al. 1980, Stolz \& Adams 1999, Domaradzki \& Adams 2002), which are based on reconstructing the SGS stress to account for backscattering effects. Balarac et al. (2013) developed a dynamic regularized gradient model, which is able to better predict space-time correlations compared with eddy-viscosity SGS models and gradient SGS models.

LaBryer et al. (2015) proposed a spatiotemporal filtering approach for improving the LES prediction of the underlying spatiotemporal structures of turbulent flows. In this approach, a spatial-temporal filter is applied to the Navier-Stokes equations, and the resulting SGS acceleration, diffusion, and stress are modeled using mean square error minimization and conditional stochastic estimations (Langford \& Moser 1999). For Burgers turbulence, spatiotemporal filtering better predicts the modal time correlations than does either spatial or temporal filtering alone. In early studies, Pruett et al. (2003) developed a temporal filtering approach to formally link LES with DNS and RANS. The temporal filters can be combined with an appropriate spatial filter to achieve Galilean invariance. This temporal LES is capable of predicting both the frequency spectra of the Burgers equations and the main characteristics of turbulent channel flows (Fadai-Ghotbi et al. 2010).

The effects of SGS modeling on sound radiation in LES can be studied through space-time correlations. In terms of the Lighthill acoustic analogy, the far-field sound is explicitly dependent on the space-time correlations of turbulent sound sources. Hence, the effects of SGS models on sound radiation can be estimated using space-time correlations. Rubinstein \& Zhou (2002) used space-time correlation models to estimate the SGS contributions to sound power spectra. Their results show that sound power spectra at high frequencies are significantly underestimated if the SGS motions are simply ignored. He et al. (2004) found that the larger decorrelation timescales 
obtained using eddy-viscosity SGS models would result in significant reductions of acoustic power spectra at higher frequencies. Both those errors exist in LES and in combination result in the left shifts of the spectral peaks to low frequencies. The results obtained from space-time correlation models are consistent with the assessment of LES-based sound radiation calculations. The SGS contribution cannot simply be neglected, and the SGS models are required to recover the acoustic intensity and adjust the locations of the peaks in the spectra (Bailly et al. 1997, Seror et al. 2000). In early studies, Bodony \& Lele (2003) highlighted the important contributions of missing scales to sound radiation and proposed an SGS noise model for them. Recently, a kinematic simulation was used to reconstruct the missing-scale motions in isotropic turbulence (Fung et al. 1992, Yao \& He 2009) and turbulent shear flows (Ghate \& Lele 2015).

Several models for space-time correlations in the framework of the Lighthill acoustic analogy have been developed. The characteristics of space-time correlation models are critically important to sound generation and radiation (Viswanathan et al. 2011). For sound radiated from isotropic turbulence, if the random sweeping model for space-time correlations is invoked, the frequency spectra scale as $-4 / 3$; if the local straining model is used instead, the scaling is $-7 / 2$ (Rubinstein \& Zhou 2000). Lilley (1994) assumed that space-time correlations are separable into space and time variables, where the spatial correlations are modeled as Gaussian functions and the time correlations are modeled as exponential functions, in accordance with the time correlations in DNS. For jet noise, the space-time correlation model is generalized in the fixed and moving frames and fitted with experimental and DNS data (Bailly et al. 1997, Morris \& Farassat 2002). To identify the sources of jet noise, Tam \& Auriault (1999) and Tam et al. (2005) developed a model that can match the envelopes of space-time correlations in a moving frame. During the development of a hybrid source model for predicting high-speed jet noise, Leib \& Goldstein (2011) proposed a nonseparable space-time correlation model in the moving frame.

\subsection{Lagrangian Time Correlations for Large-Eddy Simulations of Particle-Laden Turbulence}

In LES of particle-laden turbulence, large-scale fluid velocities are resolved with an appropriate SGS model, and the resolved velocities are then substituted into the particle equations of motion to calculate particle trajectories. Due to the chaotic motions of particles in turbulent flows, it is unlikely that LES could exactly track the particle trajectories. Instead, it is expected that LES can predict the Lagrangian statistics of particle motions, typically Lagrangian time correlations. However, the current LES suffers from two fundamental challenges. First, the SGS velocity models may not fully reproduce the effects of SGS velocities on the resolved velocity fields and could modify the Lagrangian statistics of the resolved velocity fields. Second, the SGS velocity fields mainly determine the motions of particle pairs with separation distances below the resolved scales; thus, the omission of SGS velocities may significantly change the particle dispersion and clustering. Therefore, SGS particle models are needed to address these challenges.

Yang et al. (2008) investigated the effects of SGS modeling on the Lagrangian statistics of fluid particles in isotropic and homogeneous turbulence using DNS and LES with a spectral eddyviscosity model. LES is capable of predicting the Lagrangian statistics of single fluid particles because they are dominated by large-scale motions. However, LES significantly underestimates the relative dispersion rates of particle pairs and multiple particles for separation distances that are smaller than or comparable to the minimum resolvable scales. The closure arguments show that the spectral eddy-viscosity SGS model increases the timescales of Lagrangian time correlations, leading to the slower decay of Lagrangian space-time correlations. This is similar to the effects of the spectral eddy-viscosity SGS model on Eulerian space-time correlations (He et al. 2002): The eddy-viscosity SGS models increase the time correlations. 


\section{SUBGRID-SCALE MOTION, LAGRANGIAN TIME CORRELATION, AND SUBGRID-SCALE PARTICLE MODELING}

Subgrid-scale motion mainly determines the dispersion rates of particle pairs with separation distances that are smaller than or comparable to the minimum resolvable scales. The absence of subgrid-scale motion leads to the slower dispersion of particle pairs. This can be found by comparing filtered velocities with unfiltered ones: Lagrangian time correlations from filtered velocities decay much slower than ones from unfiltered velocities. Therefore, an SGS particle model is needed to account for the effects of the subgrid-scale motions on particle motions.

The effects of SGS motion on inertial particles have been examined by comparing data from DNS and filtered direct numerical simulation (FDNS) (Fede \& Simonin 2006, Pozorski \& Apte 2009) (see the sidebar Subgrid-Scale Motion, Lagrangian Time Correlation, and Subgrid-Scale Particle Modeling). Early studies showed that one-particle statistics are slightly affected by SGS motions and can be predicted by LES (Wang \& Squires 1996, Armenio et al. 1999). In contrast, the collision-related statistics of inertial particles can be significantly affected by SGS motions: For small Stokes numbers, FDNS underpredicts the radial distribution function and radial relative velocity at contact; for intermediate Stokes numbers, FDNS results are larger than those from DNS; and for larger Stokes numbers, the collision rate can be well predicted by FDNS (Jin et al. 2010, Ray \& Collins 2011). For practical LES, the errors due to neglecting the SGS motions are combined with those caused by the SGS model, which lead to profound effects on the collisionrelated statistics of inertial particles (Jin et al. 2010, Chibbaro et al. 2014, Cernick et al. 2015).

Figure $2 \boldsymbol{a}, \boldsymbol{b}$ shows the particle distribution for $S t_{K}=1.0$ superimposed on the vorticity contours from DNS and LES with a spectral eddy-viscosity model. Evidently, the particles accumulate in regions of low vorticity and high strain rates (Squires \& Eaton 1991, Wang \& Maxey 1993, Balachandar \& Eaton 2010). However, the opposite effects of the SGS motions on particle clustering are found at different Stokes numbers: The particle clustering is greater in DNS than in LES at small Stokes numbers; in contrast, the particle distribution is less uniform in DNS compared to LES at large Stokes numbers (not shown in figure) (Pozorski \& Apte 2009, Jin et al. 2010, Ray \& Collins 2011). Figure $2 c$ plots the particle density spectra obtained from DNS, FDNS, and LES. The FDNS result is in between those of the DNS and LES. The peak from the LES is located to the left of the one from the DNS, and the variances (area integrations) from the LES and FDNS are smaller than that from the DNS (Jin et al. 2010, Urzay et al. 2014). These results indicate that the particles in the LES and FDNS are located at larger scales and are more uniform than those in DNS at small Stokes numbers.

Ray \& Collins (2011) demonstrated that a simple rescaling of the Stokes numbers based on the timescales of the resolved velocity could not reproduce the Lagrangian statistics of the particle motions because there is nonlinear coupling between the particle velocities and positions. The approximate deconvolution method could recover the one-particle statistics and, to some extent, improve the prediction of two-particle statistics with separations approximately equal to the grid resolution (Kuerten \& Vreman 2005, Kuerten 2006, Shotorban et al. 2007). In particular, the dynamic approximate deconvolution method based on elliptic differential filters could better predict the statistics of the preferential concentrations (Park et al. 2015). Stochastic Langevin equations have been used to reconstruct the SGS motions of inertial particles. If the characteristic timescales of one-particle motions are introduced, they are capable of predicting the one-particle statistics (Fede et al. 2006, Shotorban \& Mashayek 2006, Wei et al. 2006). Recently, a class of stochastic equations has been developed to predict two-particle statistics (Ray \& Collins 2013, Mazzitelli 

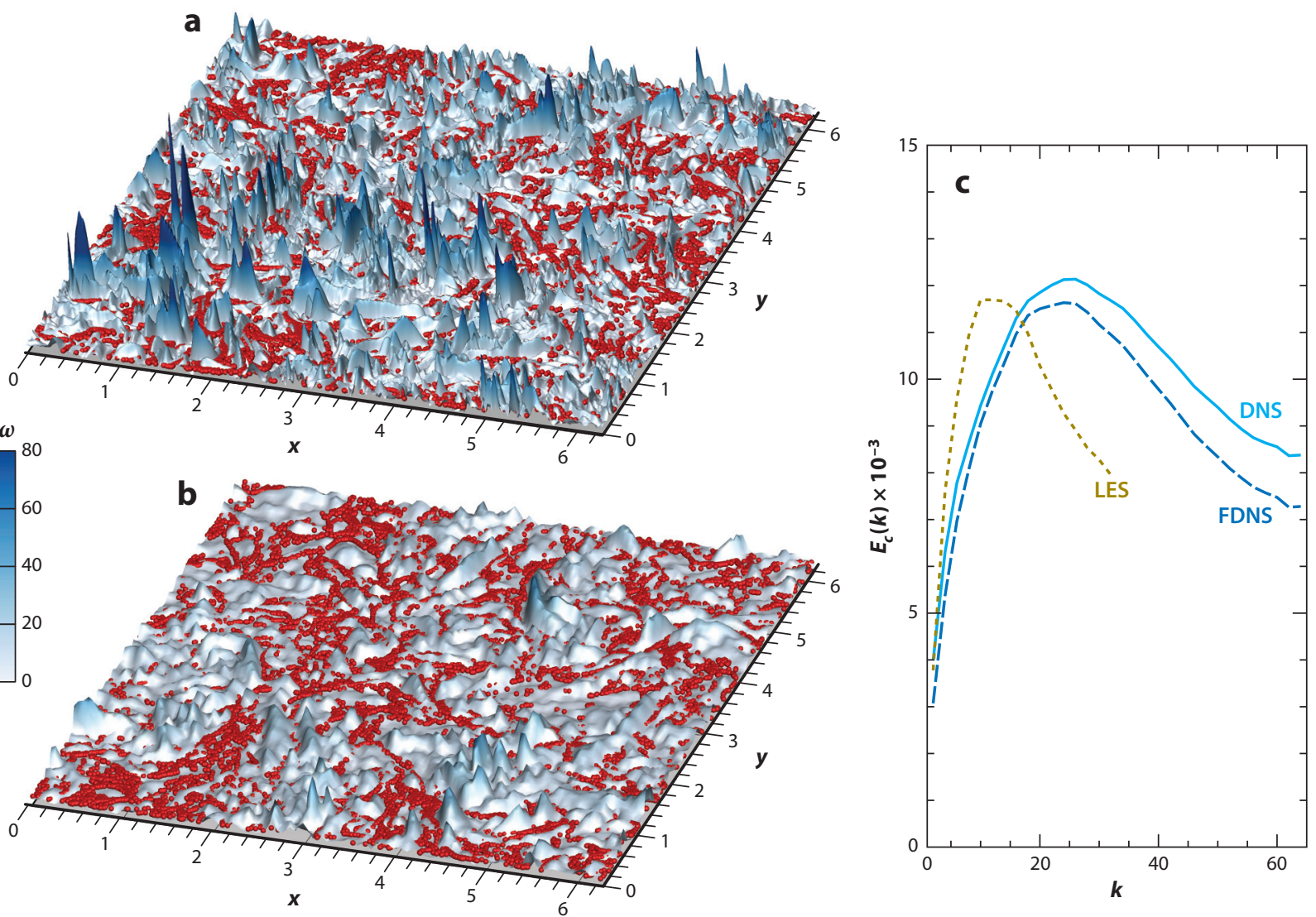

Figure 2

$(a, b)$ Slices of preferential concentrations of inertial particles at $S t_{K}=1.0$ with a thickness of $2 \eta$ overlaid on vorticity magnitude contours of isotropic turbulence from direct numerical simulation (DNS) and large-eddy simulation (LES), respectively. The slices are on the $(x, y)$ plane at the center $z=L / 2 . \eta$ is the dissipation scale. (c) Particle density spectra in DNS, filtered direct numerical simulation (FDNS), and LES.

et al. 2014). Kinematic simulations have also been used to reconstruct SGS motions, with inputs of the characteristic timescales (Flohr \& Vassilicos 2000, Jung et al. 2008), leading to a better prediction of the two-particle statistics (Ray \& Collins 2014). Pozorski \& Apte (2009) noted that a spatial filter is valid only for field variables and is not applied to any one-dimensional particle trajectory. Accordingly, Lagrangian filtering is proposed for particle motions and used to develop the Lagrangian version of the deconvolution and structural SGS model (He et al. 2012a).

\section{SUMMARY POINTS}

1. Space-time correlation models for canonical turbulent flows include the random sweeping model and local straining model for isotropic and homogeneous turbulence, Taylor's frozen-flow model and the EA model for turbulent shear flows, and the linear-wave propagation model and swept-wave model for compressible turbulence. These models describe how the spatial and temporal scales of turbulent motions are coupled and provide the characteristic timescales for two-point closure approaches. 
2. The frozen-flow hypothesis in Taylor's model omits the distortion of convected eddies, leading to a linear approximation to iso-correlation contours. The EA model accounts for the distortion of convected eddies via a second-order approximation to the iso-correlation contours. This model includes both mean-flow convection and random sweeping effects and can be used to calculate the spatial energy spectra from temporal information in turbulent shear flows.

3. Accurate predictions of space-time correlations are critically important to LES of turbulence-generated noise and particle-laden turbulence. However, the characteristic timescales of space-time correlations are overpredicted by the common class of dissipative SGS models because dissipative SGS models are based on energy balance equations and the timescales are not explicitly considered. Space-time correlations can be used as a diagnostic tool and construction approach for time-accurate SGS models.

4. The Lagrangian statistics of turbulence-driven particle motions in LES are affected by both SGS velocity models and the missing SGS flow velocities. The effects of the SGS flow velocities are significant, especially for small-Stokes number particles with separation distances that are smaller than or comparable to the minimum resolvable scales. Therefore, an SGS particle model is needed to account for the effects of the SGS velocities on particle dispersion and clustering. The approximate deconvolution method and stochastic Langevin equations are being developed for this purpose.

\section{FUTURE ISSUES}

1. Space-time correlation models for other flow features, such as pressure and concentration, must be developed, whereas space-time correlation models for velocity fluctuations remain to be improved. It is unknown whether the decorrelation mechanisms for space-time correlations remain valid for higher-order spatial and temporal statistics. Such problems might be explored using a successive approximation to iso-correlation contours starting from Taylor's frozen-flow hypothesis.

2. A substantial challenge in turbulence theory is the determination of the relationship between Eulerian and Lagrangian space-time correlations. This is related to understanding the Eulerian and Lagrangian decorrelation of small-scale eddies in turbulent flows. Lumley (1962) noted that finding such a relationship would imply that one must solve a particular closure problem.

3. It is important to develop time-accurate SGS models to predict space-time correlations. For this purpose, structural SGS models should be examined in terms of space-time correlations. Stochastic SGS models, such as random forcing models, represent alternatives that could correct the timescales of resolved velocity fields. Kinematic simulations that are limited to SGS motion (or kinematic SGS models) can be used to represent unresolved velocity fields with imposed spatial-temporal statistics.

4. Space-time correlation models are effective tools for reconstructing full space-time energy spectra from a subset of spatial and temporal signals that are now available from PIV measurements. Taylor's model represents a method for converting between spatial 
and temporal signals obtained from hot-wire measurements. These models motivate the development of data-driven learning methods for mapping a subset of a field of view to the full spectra in PIV measurements.

\section{DISCLOSURE STATEMENT}

The authors are not aware of any biases that might be perceived as affecting the objectivity of this review.

\section{ACKNOWLEDGMENTS}

G.H. thanks R. Rubinstein for stimulating discussions on space-time correlations. He is truly grateful to have the opportunity to work with J.C. Li, S.Y. Chen, and R.H. Kraichnan and acknowledges the hospitality received at the Center for Turbulence Research at Stanford University during multiple visits. The authors are grateful to J.C. Chen, C.H. Geng, and B.J. Shi for help with the figures. This work was supported by the National Natural Science Foundation of China under project numbers 11232011, 11572331, 11472277, and U1230126.

\section{LITERATURE CITED}

Armenio V, Piomelli U, Fiorotto V. 1999. Effect of the subgrid scales on particle motion. Phys. Fluids 11:3030-42

Bailly C, Lafon P, Candel S. 1997. Subsonic and supersonic jet noise predictions from statistical source models. AIAA 7. 35:1688-96

Balachandar S, Eaton JK. 2010. Turbulent dispersed multiphase flow. Annu. Rev. Fluid Mech. 42:111-33

Balarac G, Le Sommer J, Meunier X, Vollant A. 2013. A dynamic regularized gradient model of the subgridscale scalar flux for large eddy simulations. Phys. Fhids 25:075107

Bardina J, Ferziger JH, Reynolds WC. 1980. Improved subgrid-scale models for large-eddy simulation. Presented at AIAA Fluid Plasma Dyn. Conf., 13th, Snowmass, CO, AIAA Pap. 1980-1357

Bertoglio JP. 1985. A stochastic subgrid model for sheared turbulence. Lect. Notes Phys. 230:100-19

Biferale L, Boffetta G, Celani A, Devenish BJ, Lanotte A, Toschi F. 2004. Multifractal statistics of Lagrangian velocity and acceleration in turbulence. Phys. Rev. Lett. 93:064502

Bodony DJ, Lele SK. 2003. A statistical subgrid scale noise model: formulation. Presented at AIAA/CEAS Aeroacoust. Conf., 9th, Hilton Head, SC, AIAA Pap. 2003-3252

Borgas MS. 1993. The multifractal Lagrangian nature of turbulence. Philos. Trans. R. Soc. Lond. A 342:379-411

Bos WJT, Bertoglio JP. 2013. Lagrangian Markovianized field approximation for turbulence. f. Turbul. 14:99-120

Cambon C, Scott JF. 1999. Linear and nonlinear models of anisotropic turbulence. Annu. Rev. Fluid Mech. $31: 1-53$

Carati D, Ghosal S, Moin P. 1995. On the representation of backscatter in dynamic localization models. Phys. Fluids 7:606-16

Cernick MJ, Tullis SW, Lightstone MF. 2015. Particle subgrid scale modelling in large-eddy simulations of particle-laden turbulence. F. Turbul. 16:101-35

Chen SY, Kraichnan RH. 1989. Sweeping decorrelation in isotropic turbulence. Phys. Fluids A 1:2019-24

Chevillard L, Roux SG, Levêque E, Mordant N, Pinton JF, Arneodo A. 2003. Lagrangian velocity statistics in turbulent flows: effects of dissipation. Phys. Rev. Lett. 91:214502

Chibbaro S, Marchioli C, Salvetti MV, Soldati A. 2014. Particle tracking in LES flow fields: conditional Lagrangian statistics of filtering error. F. Turbul. 15:22-33 
Clark RA, Ferziger JH, Reynolds WC. 1979. Evaluation of sub-grid-scale models using an accurately simulated turbulent flow. 7. Fluid Mech. 91:1-16

Corrsin S. 1959. Progress report on some turbulent diffusion research. Adv. Geophys. 6:161-64

Davies POA, Fisher MJ, Barratt MJ. 1963. The characteristics of the turbulence in the mixing region of a round jet. F. Fluid Mech. 15:337-67

de Kat R, Ganapathisubramani B. 2015. Frequency-wavenumber mapping in turbulent shear flows. F. Fluid Mech. 783:166-90

del Álamo JC, Jiménez J. 2009. Estimation of turbulent convection velocities and corrections to Taylor's approximation. 7. Fluid Mech. 640:5-26

Domaradzki JA, Adams NA. 2002. Direct modelling of subgrid scales of turbulence in large eddy simulations. 7. Turbul. $3: \mathrm{N} 24$

Dong YH, Sagaut P. 2008. A study of time correlations in lattice Boltzmann-based large-eddy simulation of isotropic turbulence. Phys. Fluids 20:035105

Fadai-Ghotbi A, Friess C, Manceau R, Gatski TB, Borée J. 2010. Temporal filtering: a consistent formalism for seamless hybrid RANS-LES modeling in inhomogeneous turbulence. Int. F. Heat Fluid Flow 31:378-89

Favier B, Godeferd FS, Cambon C. 2010. On space and time correlations of isotropic and rotating turbulence. Phys. Fluids 22:015101

Fede P, Simonin O. 2006. Numerical study of the subgrid fluid turbulence effects on the statistics of heavy colliding particles. Phys. Fluids 18:045103

Fede P, Simonin O, Villedieu P, Squires KD. 2006. Stochastic modeling of the turbulent subgrid fluid velocity along inertial particle trajectories. Proc. Summer Program 2006, pp. 247-58. Stanford, CA: Stanford Univ. Cent. Turbul. Res.

Flohr P, Vassilicos JC. 2000. A scalar subgrid model with flow structure for large-eddy simulations of scalar variances. 7. Fluid Mech. 407:315-49

Fox RO. 2012. Large-eddy-simulation tools for multiphase flows. Annu. Rev. Fluid Mech. 44:47-76

Frisch U. 1995. Turbulence: The Legacy of A.N. Kolmogorov. Cambridge, UK: Cambridge Univ. Press

Fung JCH, Hunt JCR, Malik NA, Perkins RJ. 1992. Kinematic simulation of homogeneous turbulence by unsteady random Fourier modes. F. Fluid Mech. 236:281-318

Geng CH, He GW, Wang YS, Xu CX, Lozano-Duran A, Wallace JM. 2015. Taylor's hypothesis in turbulent channel flow considered using a transport equation analysis. Phys. Fluids 27:025111

Ghate AS, Lele SK. 2015. A modeling framework for wind farm analysis: wind turbine wake interactions. Presented at AIAA SciTech 2015, Wind Energy Symp., 33rd, Kissimmee, FL, AIAA Pap. 2015-0725

Gotoh T, Rogallo RS, Herring JR, Kraichnan RH. 1993. Lagrangian velocity correlations in homogeneous isotropic turbulence. Phys. Fluids A 5:2846-64

Grossmann S, Lohse D. 2001. Thermal convection for large Prandtl numbers. Phys. Rev. Lett. 86:3316-19

Guo L, Li D, Zhang X, He GW. 2012. LES prediction of space-time correlations in turbulent shear flows. Acta Mech. Sin. 28:993-98

He GW, Ansari HP, Jin GD, Mani A. 2012a. A Lagrangian filtering approach for large-eddy simulation of particle-laden turbulence. Proc. Summer Program 2012, pp. 375-83. Stanford, CA: Stanford Univ. Cent. Turbul. Res.

He GW, Jin GD, Zhao X. 2009. Scale-similarity model for Lagrangian velocity correlations in isotropic and stationary turbulence. Phys. Rev. E 80:066313

He GW, Rubinstein R, Wang LP. 2002. Effects of subgrid-scale modeling on time correlations in large eddy simulation. Phys. Fluids 14:2186-93

He GW, Wang M, Lele SK. 2004. On the computation of space-time correlations by large-eddy simulation. Phys. Fluids 16:3859-67

He GW, Zhang JB. 2006. Elliptic model for space-time correlations in turbulent shear flows. Phys. Rev. E 73:055303

He XZ, Bodenschatz E, Ahlers G. 2016. Azimuthal diffusion of the large-scale-circulation plane, and absence of significant non-Boussinesq effects, in turbulent convection near the ultimate-state transition. 7 . Fluid Mech. 791:R3-13

He XZ, Funfschilling D, Nobach H, Bodenschatz E, Ahlers G. 2012b. Transition to the ultimate state of turbulent Rayleigh-Bénard convection. Phys. Rev. Lett. 108:024502 
He XZ, He GW, Tong P. 2010. Small-scale turbulent fluctuations beyond Taylor's frozen-flow hypothesis. Phys. Rev. E 81:065303

He XZ, van Gils DPM, Bodenschatz E, Ahlers G. 2014. Logarithmic spatial variations and universal $f^{-1}$ power spectra of temperature fluctuations in turbulent Rayleigh-Bénard convection. Phys. Rev. Lett. 112:174501

He XZ, van Gils DPM, Bodenschatz E, Ahlers G. 2015. Reynolds numbers and the elliptic approximation near the ultimate state of turbulent Rayleigh-Bénard convection. New 7. Phys. 17:063028

Heskestad G. 1965. A generalized Taylor hypothesis with application for high Reynolds number turbulent shear flows. 7. Appl. Mech. 32:735-39

Hogg J, Ahlers G. 2013. Reynolds-number measurements for low-Prandtl-number turbulent convection of large-aspect-ratio samples. F. Fluid Mech. 725:664-80

Homann H, Kamps O, Friedrich R, Grauer R. 2009. Bridging from Eulerian to Lagrangian statistics in 3D hydro- and magnetohydrodynamic turbulent flows. New F. Phys. 11:073020

Jin GD, He GW, Wang LP. 2010. Large-eddy simulation of turbulent collision of heavy particles in isotropic turbulence. Phys. Fluids 22:055106

Jung J, Yeo K, Lee C. 2008. Behavior of heavy particles in isotropic turbulence. Phys. Rev. E 77:016307

Kaneda Y. 1981. Renormalized expansions in the theory of turbulence with the use of the Lagrangian position function. F. Fluid Mech. 107:131-45

Kim J, Hussain F. 1993. Propagation velocity of perturbations in turbulent channel flow. Phys. Fluids A 5:695-706

Kraichnan RH. 1959. The structure of isotropic turbulence at very high Reynolds numbers. F. Fluid Mech. 5:497-543

Kraichnan RH. 1962. Turbulent thermal convection at arbitrary Prandtl number. Phys. Fluids 5:1374-89

Kraichnan RH. 1964. Kolmogorov hypotheses and Eulerian turbulence theory. Phys. Fluids 7:1723-34

Kraichnan RH. 1968. Lagrangian-history statistical theory for Burgers equation. Phys. Fluids 11:265-77

Kraichnan RH. 1977. Lagrangian velocity covariance in helical turbulence. 7. Fluid Mech. 81:385-98

Kuerten JGM. 2006. Subgrid modeling in particle-laden channel flow. Phys. Fluids 18:025108

Kuerten JGM, Vreman AW. 2005. Can turbophoresis be predicted by large-eddy simulation? Phys. Fluids 17:011701

LaBryer A, Attar PJ, Vedula P. 2015. A framework for large eddy simulation of Burgers turbulence based upon spatial and temporal statistical information. Phys. Fluids 27:035116

Langford JA, Moser RD. 1999. Optimal LES formulations for isotropic turbulence. 7. Fluid Mech. 398:321-46

Lee S, Lele SK, Moin P. 1992. Simulation of spatially evolving turbulence and the applicability of Taylor's hypothesis in compressible flow. Phys. Fluids A 4:1521-30

Leib SJ, Goldstein ME. 2011. Hybrid source model for predicting high-speed jet noise. AIAA 7. 49:1324-35

Leith CE. 1990. Stochastic backscatter in a subgrid-scale model: plane shear mixing layer. Phys. Fluids $A$ 2:297-99

Li D, Zhang X, He GW. 2013. Temporal decorrelations in compressible isotropic turbulence. Phys. Rev. E 88:021001

Lighthill MJ. 1952. On sound generated aerodynamically. I. General theory. Proc. R. Soc. Lond. A 211:564-87

Lilley GM. 1994. The radiated noise from isotropic turbulence. Theor. Comput. Fluid Dyn. 6:281-301

Lin CC. 1953. On Taylor's hypothesis and the acceleration terms in the Navier-Stokes equations. Q. Appl. Math. 10:295-306

Lohse D, Xia KQ. 2010. Small-scale properties of turbulent Rayleigh-Bénard convection. Annu. Rev. Fluid Mech. 42:335-64

Loucks RB, Wallace JM. 2012. Velocity and velocity gradient based properties of a turbulent plane mixing layer. 7. Fluid Mech. 699:280-319

Lumley JL. 1962. The mathematical nature of the problem of relating Langrangian and Eulerian statistical functions in turbulence. In Mécanique de la Turbulence, pp. 17-26. Paris: CNRS

Lumley JL. 1965. Interpretation of time spectra measured in high-intensity shear flows. Phys. Fluids 8:1056-62

Marstorp L, Brethouwer G, Johansson, AV. 2007. A stochastic subgrid model with application to turbulent flow and scalar mixing. Phys. Fluids 19:035107

Martin MP. 2005. Preliminary study of the SGS time scales for compressible boundary layers using DNS data. Presented at AIAA Aerosp. Sci. Meet. Exhib., 43rd, Reno, NV, AIAA Pap. 2005-0665 
Mason PJ, Thomson DJ. 1992. Stochastic backscatter in large-eddy simulations of boundary layers. F. Fluid Mech. 242:51-78

Mazzitelli IM, Toschi F, Lanotte AS. 2014. An accurate and efficient Lagrangian sub-grid model. Phys. Fluids 26:095101

Meneveau C, Katz J. 2000. Scale-invariance and turbulence models for large-eddy simulation. Annu. Rev. Fluid Mech. 32:1-32

Moin P. 2009. Revisiting Taylor's hypothesis. 7. Fluid Mech. 640:1-4

Morris PJ, Farassat F. 2002. Acoustic analogy and alternative theories for jet noise prediction. AIAA $\mathcal{F}$. 40:671-80

O’Brien J, Urzay J, Ihme M, Moin P, Saghafian A. 2014. Subgrid-scale backscatter in reacting and inert supersonic hydrogen-air turbulent mixing layers. F. Fluid Mech. 743:554-84

Orszag SA. 1977. Lectures on the statistical theory of turbulence. In Fluid Dynamics: Lecture Notes of the Les Houches Summer School 1973, ed. R Balian, JL Peube, pp. 235-374. New York: Gordon \& Breach

Park GI, Urzay J, Bassenne M, Moin P. 2015. A dynamic subgrid-scale model based on differential filters for LES of particle-laden turbulent flows. In Annu. Res. Briefs 2015, pp. 17-26. Stanford, CA: Stanford Univ. Cent. Turbul. Res.

Park N, Yoo JY, Choi H. 2005. Toward improved consistency of a priori tests with a posteriori tests in large eddy simulation. Phys. Fluids 17:015103

Piomelli U, Balint JL, Wallace JM. 1989. On the validity of Taylor's hypothesis for wall-bounded flows. Phys. Fluids A 1:609-11

Piomelli U, Cabot WH, Moin P, Lee SS. 1991. Subgrid-scale backscatter in turbulent and transitional flows. Phys. Fluids A 3:1766-71

Pozorski J, Apte SV. 2009. Filtered particle tracking in isotropic turbulence and stochastic modeling of subgridscale dispersion. Int. F. Multiphase Flow 35:118-28

Pruett CD, Gatski TB, Grosch CE, Thacker WD. 2003. The temporally filtered Navier-Stokes equations: properties of the residual stress. Phys. Fluids 15:2127-40

Ray B, Collins LR. 2011. Preferential concentration and relative velocity statistics of inertial particles in Navier-Stokes turbulence with and without filtering. F. Fluid Mech. 680:488-510

Ray B, Collins LR. 2013. Investigation of sub-Kolmogorov inertial particle pair dynamics in turbulence using novel satellite particle simulations. F. Fluid Mech. 720:192-211

Ray B, Collins LR. 2014. A subgrid model for clustering of high-inertia particles in large-eddy simulations of turbulence. 7. Turbul. 15:366-85

Renard N, Deck S. 2015. On the scale-dependent turbulent convection velocity in a spatially developing flat plate turbulent boundary layer at Reynolds number $\operatorname{Re}_{\theta}=13000$. F. Fluid Mech. 775:105-48

Rubinstein R, Zhou Y. 1999. Effects of helicity on Lagrangian and Eulerian time correlations in turbulence. Pbys. Fluids 11:2288-90

Rubinstein R, Zhou Y. 2000. The frequency spectrum of sound radiated by isotropic turbulence. Phys. Lett. A 267:379-83

Rubinstein R, Zhou Y. 2002. Characterization of sound radiation by unresolved scales of motion in computational aeroacoustics. Eur. F. Mech. B 21:105-11

Seror C, Sagaut P, Bailly C, Juve D. 2000. Subgrid-scale contribution to noise production in decaying isotropic turbulence. AIAA 7. 38:1795-803

Shotorban B, Mashayek F. 2006. A stochastic model for particle motion in large-eddy simulation. F. Turbul. 7:N18

Shotorban B, Zhang KKQ, Mashayek F. 2007. Improvement of particle concentration prediction in large-eddy simulation by defiltering. Int. F. Heat Mass Transfer 50:3728-39

Smith FB, Hay JS. 1961. The expansion of clusters of particles in the atmosphere. Q. 7. R. Meteorol. Soc. $87: 82-101$

Smith LM, Woodruff SL. 1998. Renormalization-group analysis of turbulence. Annu. Rev. Fluid Mech. 30: 275-310

Smits AJ, McKeon BJ, Marusic I. 2011. High-Reynolds number wall turbulence. Annu. Rev. Fluid Mech. $43: 353-75$ 
Squires KD, Eaton JK. 1991. Preferential concentration of particles by turbulence. Phys. Fluids A 3:1169-79

Stolz S, Adams NA. 1999. An approximate deconvolution procedure for large-eddy simulation. Phys. Fluids 11:1699-701

Tam CKW, Auriault L. 1999. Jet mixing noise from fine-scale turbulence. AIAA 7. 37:145-53

Tam CKW, Pastuchenko NN, Viswanathan K. 2005. Fine-scale turbulence noise from hot jets. AIAA F. 43:1675-83

Taylor GI. 1938. The spectrum of turbulence. Proc. R. Soc. Lond. A 164:476-90

Tennekes H. 1975. Eulerian and Lagrangian time microscales in isotropic turbulence. F. Fluid Mech. 67:561-67

Tsinober A, Vedula P, Yeung PK. 2001. Random Taylor hypothesis and the behavior of local and convective accelerations in isotropic turbulence. Phys. Fluids 13:1974-84

Urzay J, Bassenne M, Park G, Moin P. 2014. Characteristic regimes of subgrid-scale coupling in LES of particle-laden turbulent flows. In Annu. Res. Briefs 2014, pp. 3-13. Stanford, CA: Stanford Univ. Cent. Turbul. Res.

Viswanathan K, Underbrink JR, Brusniak L. 2011. Space-time correlation measurements in near fields of jets. AIAA 7. 49:1577-99

Wallace JM. 2014. Space-time correlations in turbulent flow: a review. Theor. Appl. Mech. Lett. 4:022003

Wang LP, Maxey MR. 1993. Settling velocity and concentration distribution of heavy particles in homogeneous isotropic turbulence. F. Fluid Mech. 256:27-68

Wang M, Freund JB, Lele SK. 2006. Computational prediction of flow-generated sound. Annu. Rev. Fluid Mech. 38:483-512

Wang QZ, Squires KD. 1996. Large eddy simulation of particle-laden turbulent channel flow. Phys. Fluids 8:1207-23

Wang W, Guan XL, Jiang N. 2014. TRPIV investigation of space-time correlation in turbulent flows over flat and wavy walls. Acta Mech. Sin. 30:468-79

Wei GX, Vinkovic I, Shao L, Simoëns S. 2006. Scalar dispersion by a large-eddy simulation and a Lagrangian stochastic subgrid model. Phys. Fluids 18:095101

Wilczek M, Narita Y. 2012. Wave-number-frequency spectrum for turbulence from a random sweeping hypothesis with mean flow. Phys. Rev. E 86:066308

Wilczek M, Stevens RJAM, Meneveau C. 2015. Spatio-temporal spectra in the logarithmic layer of wall turbulence: large-eddy simulations and simple models. F. Fluid Mech. 769:R1-12

Wills JAB. 1964. On convection velocities in turbulent shear flows. F. Fluid Mech. 20:417-32

Xu HT, Ouellette NT, Bodenschatz E. 2006. Multifractal dimension of Lagrangian turbulence. Phys. Rev. Lett. 96:114503

Yang Y, He GW, Wang LP. 2008. Effects of subgrid-scale modeling on Lagrangian statistics in large-eddy simulation. 7. Turbul. 9:N8

Yao HD, He GW. 2009. A kinematic subgrid scale model for large-eddy simulation of turbulence-generated sound. F. Turbul. 10:N19

Zaman KBMQ, Hussain AKMF. 1981. Taylor hypothesis and large-scale coherent structures. F. Fluid Mech. 112:379-96

Zhao X, He GW. 2009. Space-time correlations of fluctuating velocities in turbulent shear flows. Phys. Rev. E 79:046316

Zhou Q, Li CM, Lu ZM, Liu YL. 2011. Experimental investigation of longitudinal space-time correlations of the velocity field in turbulent Rayleigh-Bénard convection. F. Fluid Mech. 683:94-111

Zhou Y. 2010. Renormalization group theory for fluid and plasma turbulence. Phys. Rep. 488:1-49 
An Appreciation of the Life and Work of William C. Reynolds (1933-2004)

Parviz Moin and G.M. Homsy ....

Inflow Turbulence Generation Methods

Xiaobua $W u$

Space-Time Correlations and Dynamic Coupling in Turbulent Flows

Guowei He, Guodong Fin, and Yue Yang ....

Motion of Deformable Drops Through Porous Media

Alexander Z. Zinchenko and Robert H. Davis

Recent Advances in Understanding of Thermal Expansion Effects in

Premixed Turbulent Flames

Vladimir A. Sabelnikov and Andrei N. Lipatnikov

Incompressible Rayleigh-Taylor Turbulence

Guido Boffetta and Andrea Mazzino

Cloud-Top Entrainment in Stratocumulus Clouds

Zuan Pedro Mellado

Simulation Methods for Particulate Flows and Concentrated Suspensions

Martin Maxey

From Topographic Internal Gravity Waves to Turbulence

S. Sarkar and A. Scotti

Vapor Bubbles

Andrea Prosperetti .

Anisotropic Particles in Turbulence

Greg A. Voth and Alfredo Soldati

Combustion and Engine-Core Noise

Matthias Ibme

Flow Structure and Turbulence in Wind Farms

Richard 7.A.M. Stevens and Charles Meneveau 
Particle Migration due to Viscoelasticity of the Suspending Liquid, and Its Relevance in Microfluidic Devices

Gaetano D'Avino, Francesco Greco, and Pier Luca Maffettone

341

Uncertainty Quantification in Aeroelasticity

Philip Beran, Bret Stanford, and Christopher Schrock 361

Model Reduction for Flow Analysis and Control

Clarence W. Rowley and Scott T.M. Dawson

Physics and Measurement of Aero-Optical Effects: Past and Present

Eric 7. Fumper and Stanislav Gordeyev

Blood Flow in the Microcirculation

Timotby W. Secomb

Impact on Granular Beds

Devaraj van der Meer....

The Clustering Instability in Rapid Granular and Gas-Solid Flows

William D. Fullmer and Christine M. Hrenya

Phoretic Self-Propulsion

Feffrey L. Moran and Fonathan D. Posner

Recent Developments in the Fluid Dynamics of Tropical Cyclones

Michael T. Montgomery and Roger K. Smith

Saph and Schoder and the Friction Law of Blasius

Paul Steen and Wilfried Brutsaert

\section{Indexes}

Cumulative Index of Contributing Authors, Volumes 1-49 ...................... 583

Cumulative Index of Article Titles, Volumes 1-49 ........................... 593

Errata

An online log of corrections to Annual Review of Fluid Mechanics articles may be found at http://www.annualreviews.org/errata/fluid 